\title{
IRRADIAÇÃO DE POLÍMEROS COM FEIXE DE ELÉTRONS: CARACTERIZAÇÃO DE ÍONS POSITIVOS ATRAVÉS DA TÉCNICA DE TEMPO-DE-VÔO
}

\author{
Maria Luiza Miranda Rocco*, Frederico Celestino Pontes, Gustavo Sebastian Faraudo e Gerardo Gerson Bezerra de \\ Souza \\ Departamento de Físico-Química, Instituto de Química, Universidade Federal do Rio de Janeiro, Cidade Universitária, \\ Ilha do Fundão, 21949-900 Rio de Janeiro - RJ \\ Daniel Eduardo Weibel \\ Facultad de Ciencias Químicas, Universidad Nacional de Córdoba, Ciudad Universitaria, 5000 Córdoba - Argentina \\ Roberto Rosas Pinho \\ Departamento de Física, Instituto de Ciências Exatas, Universidade Federal de Juiz de Fora, Campus Universitário, \\ 36036-330 Juiz de Fora - MG
}

Recebido em 25/10/02; aceito em 20/8/03

\begin{abstract}
IRRADIATION OF POLYMERS USING ELECTRON BEAMS: CHARACTERIZATION OF POSITIVE IONS THROUGH THE TIME-OF-FLIGHT TECHNIQUE. With the aim of studying the interaction of fast electrons with solid surfaces we have developed an experimental set-up based on electron stimulated desorption (ESD) coupled to time-of-flight (TOF) mass spectrometry. Poly(methyl methacrylate) and poly(vynil chloride) samples have been irradiated by a pulsed electron beam of $1.2 \mathrm{keV}$ and 0.18 $\mu$ FWHM. The results show that $\mathrm{H}^{+}$is the main ionic species to desorb after electron bombardment. In addition, other ionic fragments were also observed and assigned. These results show the potentiality of this technique in the study of ESD of polymers.
\end{abstract}

Keywords: electron stimulated desorption; time-of-flight spectrometer; polymers.

\section{INTRODUÇÃO}

Para os pesquisadores que atuam na área de Ciência de Superfícies é muito importante obter um conhecimento elementar da superfície em estudo e mais importante ainda é o conhecimento das espécies moleculares, que estão na superfície. Uma das técnicas mais poderosas de análise superficial é a Espectroscopia Auger (AES "Auger Electron Spectroscopy") ${ }^{1}$. Esta técnica fornece informação elementar, com exceção do hidrogênio e do hélio. Todavia, ela não fornece informação em relação ao estado químico das espécies adsorvidas na superfície, ou seja, espécies moleculares na superfície não podem ser detectadas. Outra técnica que fornece informação química direta sobre a superfície é a dessorção induzida por laser utilizando uma matriz especial, que absorve a radiação laser (MALDI - "Matrix-Assisted Laser Desorption Ionization") $)^{2-4}$. Apesar de ser possível atualmente fazer uma varredura do laser pela superfície com resoluções laterais da ordem de mícrons ${ }^{4}$, a técnica necessita de uma matriz, o que elimina a possibilidade de se estudar amostras in situ, além de ser altamente destrutiva. Uma das técnicas que mais se desenvolveu nos últimos 20 anos na área de análise de superfícies é a técnica de espectrometria de massas de íons secundários formados a partir do impacto de íons primários monoatômicos ou poliatômicos com a superfície (SSIMS - "Static Secondary Ion Mass Spectrometry") ${ }^{5-7}$. Esta técnica pode fornecer informação química de monocamadas adsorvidas sobre todos os tipos de superfícies e pode vir a ser a técnica preferida para análise química superficial em um futuro próximo. Entretanto, esta técnica também apresenta desvantagens: o baixo rendimento dos íons secundários, o efeito da matriz e as grandes seções de choque de dano, que limitam o uso da técnica abaixo do limite estático ${ }^{7}$, onde uma corrente de íons primários muito baixa tem que ser usada (nunca maior que $10^{13}$ íns. $\mathrm{cm}^{-2}$ ), com a

*e-mail: luiza@iq.ufrj.br finalidade de garantir que menos de $1 \%$ dos átomos ou moléculas superficiais recebam um impacto iônico.

A utilização de elétrons rápidos apresenta uma série de vantagens com respeito às técnicas analíticas mencionadas acima. Em primeiro lugar, o dano produzido por elétrons nas amostras é muito menor que aquele decorrente de um laser ou de um íon de alta energia $^{8-9}$. Segundo, é de se esperar que o efeito de matriz seja menor que no caso de SSIMS, devido à menor energia utilizada e ao menor tamanho da partícula carregada (primária) usada. Terceiro, o tamanho do feixe de elétrons pode ser, pelo menos, uma ordem de grandeza menor que o tamanho do feixe de íons. Atualmente, conseguem-se resoluções laterais de $100 \mathrm{~nm}$ com $\mathrm{Ga}^{+}$como íon primário e com $25 \mathrm{keV}$ de energia cinética ${ }^{10}$. Finalmente, e não de menor importância, pode-se obter informação química superficial com o uso de elétrons como fonte primária de excitação, conseguindo-se inclusive detectar hidrogênio, difícil de ser observado por outras técnicas.

O uso de elétrons para produzir dessorção de íons e espécies neutras é uma área muito conhecida e muito desenvolvida nos últimos 30-40 anos. Estudos de dessorção induzida por transições eletrônicas (DIET - "Desorption Induced by Electronic Transitions") mediante a utilização tanto de elétrons como de outras fontes de excitação, como laser, radiação síncrotron, etc., conseguiram finalmente obter um conhecimento suficiente para compreender os mecanismos que originam a dessorção de espécies neutras e iônicas nas faixas de excitação de valência e de camada interna. O emprego de elétrons em técnicas como SEM ("Scanning Electron Microscopy"), EMPA ("Electron MicroProbe Analysis), etc. para estudos morfológicos de superfícies é hoje em dia realizado rotineiramente, apresentando enorme importância para áreas como a biologia, eletrônica, microfabricação, etc., onde é preciso conhecer em detalhe a morfologia superficial. Entretanto, apesar de todos os estudos realizados com elétrons, a aplicação analítica de elétrons para a análise química de superfícies combinando a espectrometria de massas com a excitação de elétrons rápidos ainda não foi muito explorada. 
Ueda e colaboradores ${ }^{12-14}$ iniciaram, recentemente, o desenvolvimento de uma nova aplicação analítica da técnica de dessorção estimulada por elétrons (ESD - "Electron Stimulated Desorption"), que eles denominaram de "scanning-ESD", para a detecção de hidrogênio em superfícies, principalmente contendo $\mathrm{SiO}_{2}$ e $\mathrm{Si}$, utilizando um canhão de elétrons pulsado. O êxito da aplicação de ESDTOF para este caso particular abre novos caminhos para um uso mais geral da técnica de ESD.

Além disso, o estudo da interação de elétrons e fótons com polímeros é de fundamental importância, contribuindo decisivamente para a compreensão dos mecanismos de degradação destes materiais por radiações, de forma geral. Processos litográficos, utilizados na fabricação de micro-dispositivos mecânicos e eletrônicos ${ }^{15}$, por exemplo, envolvem a utilização de feixes de partículas de alta energia (elétrons, fótons, íons) e "resists" (polímeros). Podemos citar, nesse contexto, o processo LIGA $^{16,17}$ que emprega, geralmente, o polimetacrilato de metila (PMMA) como "resist" positivo (polímero cuja solubilidade cresce com a exposição à radiação).

Por estas razões, iniciamos recentemente o estudo de polímeros ${ }^{18}$ e outras amostras sólidas mediante a excitação pulsada com elétrons de energia controlada, juntamente com a análise por espectrometria de massas dos íons secundários formados após impacto de elétrons. Para tal, desenvolvemos um sistema experimental baseado no emprego de um espectrômetro de tempo-de-vôo (TDV). Neste sistema, um feixe de elétrons incide sobre a superfície de uma amostra sólida e como resultado diferentes fragmentos (espécies neutras, íons positivos e negativos) são formados. Os íons positivos são analisados segundo suas relações massa/carga por espectrometria de massa, mediante o emprego de um conversor tempo-digital ("time to digital converter", TDC). Na medida dos tempos-de-vôo, o sinal de parada ("stop") é fornecido pelos íons positivos e o de inicialização ("start") pelo gerador de pulsos, que controla o canhão de elétrons. Apresentamos, a seguir, alguns dos resultados iniciais, associados com a irradiação de amostras de PMMA e de cloreto de polivinila (PVC) com feixe de elétrons de 1,2 keV. Estes resultados mostraram espectros TOF característicos de cada um dos polímeros estudados.

\section{PARTE EXPERIMENTAL}

O sistema experimental baseia-se no emprego da técnica de tempo-de-vôo no estudo da dessorção iônica induzida em amostras sólidas através da interação com feixe de elétrons pulsado. Seus principais componentes são: a) câmara de vácuo; b) canhão de elétrons de energia variável $(0,1-3 \mathrm{keV})$; c) manipulador de amostras, o qual permite movimentação em torno de três eixos perpendiculares, $x, y$ e $\mathrm{z}$, além de rotação ao longo do eixo z; d) espectrômetro de massa do tipo tempo-de-vôo (TDV), desenvolvido em nosso laboratório ${ }^{19}$; e) espectrômetro de massas, comercial, do tipo quadrupolar, que varre até 200 u.m.a. (Stanford Research Systems, modelo RGA 200), para monitoramento dos gases residuais presentes na câmara de vácuo. A pressão final na câmara de trabalho é da ordem de $10^{-9}$ Torr, obtida através de um sistema constituído por uma bomba mecânica e uma bomba turbomolecular de alta capacidade (900 l/s, Alcatel). O sistema experimental pode facilmente ser adaptado para estudos envolvendo fontes de luz, tais como uma lâmpada de descarga em hélio, que emite as linhas ressonantes de hélio I $(21,21 \mathrm{eV})$ e hélio II $(40,8$ eV) ou a radiação síncrotron.

Os alvos utilizados consistiram de amostras poliméricas de espessura da ordem de $20 \mu \mathrm{m}$, preparadas com a técnica de "casting", que consiste em gotejar a solução com o polímero em suportes de alumínio, deixando-se o solvente evaporar. Os suportes, por sua vez, foram adaptados ao manipulador X-Y-Z e os polímeros irradiados com um feixe de elétrons pulsado com uma largura à meia altura do pulso
(FWHM) de 0,18 $\mu$ s e com uma freqüência de $3 \mathrm{kHz}$. O feixe de elétrons foi obtido através de um canhão de elétrons comercial, do tipo empregado em osciloscópios, de energia variável. Trabalhou-se com uma corrente da ordem de $5 \mathrm{nA} / \mathrm{cm}^{2}$, com o objetivo de minimizar efeitos de carregamento na amostra. $\mathrm{O}$ feixe de elétrons incide sobre a amostra a $30^{\circ} \mathrm{em}$ relação à normal à superfície, estando a amostra a $3,5 \mathrm{~cm}$ da entrada do espectrômetro de massa do tipo tempo-de-vôo.

$\mathrm{O}$ espectrômetro de tempo-de-vôo consiste basicamente de um sistema de extração de íons, um tubo de vôo $(25 \mathrm{~cm})$ e um detector de íons (Figura 1). Íons provenientes da amostra são acelerados em direção à primeira grade do TDV e, em seguida, atravessam uma segunda região de aceleração, antes de atingir o tubo de vôo. Utilizase ainda neste dispositivo uma lente eletrostática ${ }^{19}$ colimadora, na qual se aplica um potencial baixo, cerca de $+40 \mathrm{~V}$, para garantir a detecção eficiente de íons dotados de elevada energia cinética. $\mathrm{O}$ detector consiste de duas placas de microcanais ("microchannel plates", MCP), montadas na forma de divisa (“chevron”). Através de um programa de simulação de trajetórias de íons $\left(\mathrm{SIMION}^{20}\right)$, determinaram-se os valores iniciais para as tensões a serem aplicadas nos diversos elementos do espectrômetro (grade, lente, tubo de vôo e detector), procurando-se melhorar a resolução em massa e a eficiência de detecção. O sinal de saída do detetor foi processado mediante um sistema padrão de contagem de pulsos, constituído de um préamplificador e de um discriminador, sendo este sinal usado como o sinal de parada ("stop") da experiência. O sinal de inicialização ("start") foi obtido através de um sinal dado pelo gerador de pulsos, que controla o canhão de elétrons. Estes sinais foram enviados a um conversor tempo-digital ("time to digital converter", Schmidt Instruments, TOF2, Multistop TDC) para serem acumulados, que apresenta uma resolução máxima de $2,5 \mathrm{~ns} /$ canal.

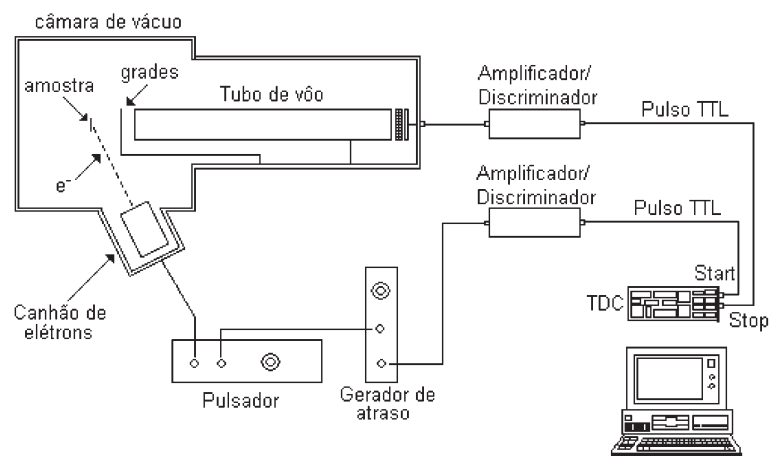

Figura 1. Diagrama do arranjo experimental utilizado no estudo da interação de elétrons com polímeros

O esquema temporal utilizado na presente experiência é mostrado na Figura 2. Para cada pulso de elétrons enviado pelo canhão para o alvo, um sinal eletrônico (sinal "start") é enviado do gerador de pulsos para o TDC. Os sinais correspondentes do detector (sinais "stop") são também enviados para o conversor, que mede os intervalos de tempo entre o sinal "start" e os correspondentes sinais "stop". Os sinais enviados pelo conversor são processados em um computador pessoal, na forma de espectro de tempo-de-vôo, que apresenta o número de contagens de cada íon detectado em função dos tempos medidos pelo conversor. Durante a experiência, o alvo ficou submetido a um potencial de extração constante de +200 V. Além disso, para evitar que o ruído gerado pelo pulsador interferisse no início dos espectros de tempo-de-vôo, um atraso de cerca de $1 \mu$ s foi aplicado entre a saída do sinal "start" do gerador de pulsos e a entrada "start" no TDC (Figura 2).

A calibração da escala de massa dos espectros obtidos foi reali- 
zada através da introdução de gases atômicos (He ou Ar) na câmara de vácuo, conforme descrito a seguir.

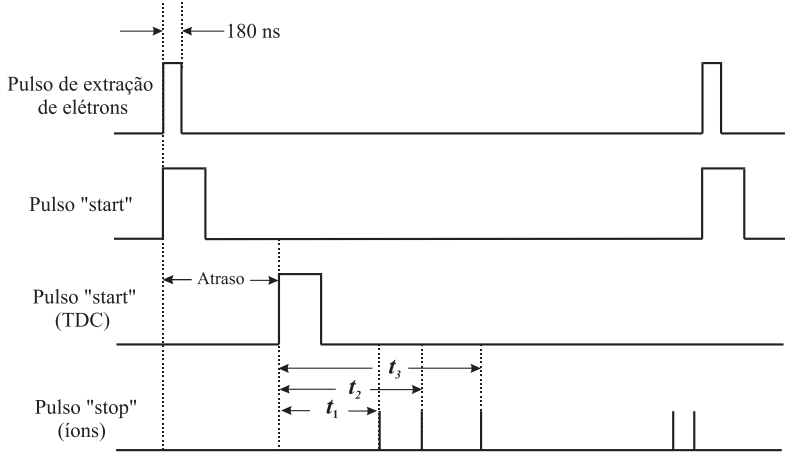

Figura 2. Diagrama de temporalização utilizado na obtenção dos espectros de tempo-de-vôo

\section{RESULTADOS E DISCUSSÃO}

\section{Calibração da escala massa/carga}

A calibração dos espectros de massa, conforme mencionado anteriormente, foi obtida através da introdução de um gás nobre (em nosso caso, hélio ou argônio) na câmara de medida, elevando-se a pressão de $10^{-9}$ para $10^{-6}-10^{-5}$ Torr. Espectros de massa do polímero bem como dos diferentes gases foram medidos simultaneamente. Maiores detalhes sobre esta técnica de calibração podem ser encontrados na referência 21. Exemplos deste procedimento de calibração para o PMMA podem ser vistos na Figura 3. Na Figura 3b, podemos observar um pico referente ao íon $\mathrm{He}^{+}$, juntamente com os picos correspondentes ao PMMA (Figura 3a). Na Figura 3c, observa-se nitidamente a presença dos íons $\mathrm{Ar}^{+}, \mathrm{Ar}^{+2}$ e $\mathrm{Ar}^{+4}$, que correspondem às relações massa/carga 40, 20 e 10, respectivamente, além dos íons referentes ao polímero. $\mathrm{O}$ pico de $\mathrm{Ar}^{+3}$ não pode ser visualizado claramente, por estar sobreposto a um pico do polímero em $5,8 \mu$ s. Além disso, uma pequena contribuição devida ao íon $\mathrm{Ar}^{+5}$ também pode ser observada no espectro da Figura 3c.

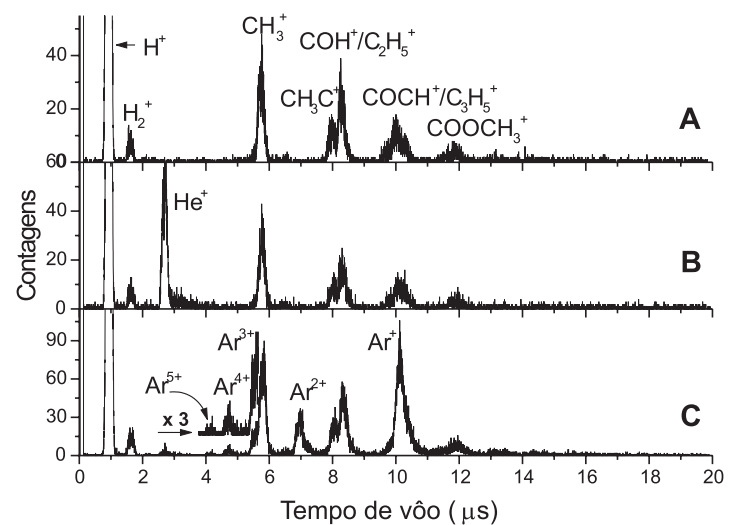

Figura 3. Espectros de tempo-de-vôo obtidos com feixe de elétrons de 1200 eV. A) PMMA; B) He (pressão 1,5 × 10-6 Torr) e PMMA; C) Ar (pressão 1,1 $\times 10^{-5}$ Torr) e PMMA

De posse destes dados e levando-se em consideração que o tempo-de-vôo para um dado íon (desde a sua saída do alvo até a chegada ao detector) é proporcional à raiz quadrada da sua razão massa/carga, isto é,

tof $=a(m / q)^{1 / 2}+b$ onde tof representa os tempos-de-vôo obtidos experimentalmente em microsegundos, $m / q$ as razões massa/carga dos íons, e $a$ e $b$ constantes relacionadas ao espectrômetro de massa do tipo TDV utilizado e às condições experimentais ${ }^{22}$, pode-se então realizar uma calibração apropriada dos espectros de tempo-de-vôo, convertendo-os em espectros de massa. Para a calibração dos espectros de massa do PMMA, os tempos-de-vôo dos íons de $\mathrm{Ar}^{+}$e $\mathrm{Ar}^{+2}$, com razão m/q de 40 e 20, respectivamente, foram utilizados na expressão acima, permitindo a determinação dos coeficientes $a$ e $b$. Trata-se de um novo e simples procedimento de calibração, que elimina a necessidade do uso de uma amostra-padrão ou de se trabalhar com diferentes valores dos potenciais de extração ${ }^{23}$.

\section{PMMA E PVC}

Um espectro de massa do PMMA, previamente calibrado, pode ser visto na Figura 4. Este espectro foi obtido para uma energia do feixe de elétrons de $1,2 \mathrm{keV}$ e cobre um intervalo de massa de até 50 u.m.a.. Diversos picos podem ser observados, sendo que o íon $\mathrm{H}^{+}$ aparece como a espécie iônica de maior intensidade, seguido do íon $\mathrm{CH}_{3}{ }^{+}$. Além disso, outros fragmentos iônicos menos intensos, tais como: $\mathrm{H}_{2}^{+} ; \mathrm{C}^{+}, \mathrm{CH}^{+}, \mathrm{CH}_{2}^{+} ; \mathrm{CH}_{3} \mathrm{C}^{+}, \mathrm{CHO}^{+}\left(\mathrm{e} / \mathrm{ou} \mathrm{C}_{2} \mathrm{H}_{5}^{+}\right) ; \mathrm{C}_{3} \mathrm{H}_{3}^{+}, \mathrm{COCH}^{+}$ (e/ou $\left.\mathrm{C}_{3} \mathrm{H}_{5}{ }^{+}\right), \mathrm{COCH}_{3}{ }^{+}\left(\mathrm{e} / \mathrm{ou} \mathrm{C}_{3} \mathrm{H}_{7}^{+}\right)$, podem também ser observados, correspondendo às razões massa/carga: 2; 12, 13, 14; 27, 29; 39, 41 e 43 , respectivamente.

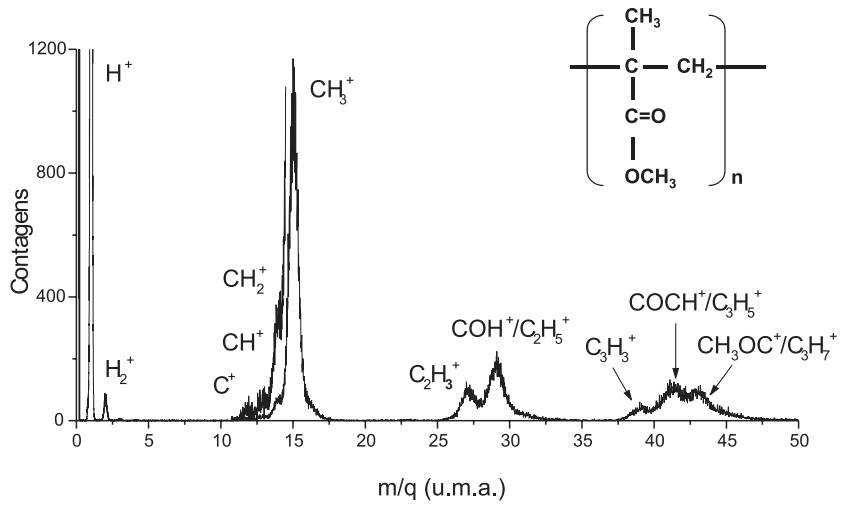

Figura 4. Espectro de massa do PMMA obtido com um feixe de elétrons de $1200 \mathrm{eV}$, cobrindo um intervalo de massa de até 50 u.m.a.

Embora não sejam apresentados, alguns picos de menor intensidade foram observados em 59, 67, 83, 86, 100 e 114 u.m.a.. Estes fragmentos provavelmente correspondem aos íons: $\mathrm{COOCH}_{3}^{+}$, $\mathrm{CH}_{2} \mathrm{CCH}_{3} \mathrm{CO}^{+}, \quad \mathrm{CH}_{2} \mathrm{CCH}_{3} \mathrm{COCH}_{2}^{+}, \quad \mathrm{CH}_{2} \mathrm{CCH}_{3} \mathrm{HCO}_{2}^{+}$, $\mathrm{CH}_{2} \mathrm{CCH}_{3} \mathrm{CO}_{2} \mathrm{CH}_{3}^{+}$(monômero) e $\mathrm{CH}_{2} \mathrm{CCH}_{3} \mathrm{CO}_{2} \mathrm{CH}_{3} \mathrm{CH}_{2}^{+}$. Para os íons mais pesados, como é esperado, domina o processo de neutralização, já que eles têm menor velocidade de dessorção quando comparados aos íons mais leves ${ }^{24}$. São, conseqüientemente, de difícil observação, requerendo potenciais de extração mais elevados e/ou tempos de aquisição mais longos.

Espectros obtidos, recentemente, com o emprego da radiação síncrotron ${ }^{23,25-28}$ apresentam os seguintes íons: $\mathrm{H}^{+}, \mathrm{CH}^{+}, \mathrm{CH}_{2}^{+}, \mathrm{CH}_{3}^{+}$, $\mathrm{CHO}^{+}$e $\mathrm{COOCH}_{3}^{+}$, quando excitados com fótons de $393 \mathrm{eV}$ e ainda o íon $\mathrm{COCH}^{+}$, para $\mathrm{h} v=560 \mathrm{eV}$. Semelhante aos resultados deste trabalho, observa-se que as espécies mais intensas correspondem aos íons $\mathrm{H}^{+}$e $\mathrm{CH}_{3}^{+}$. Acredita-se que o principal processo responsável pela dessorção iônica destes materiais, nesta região de alta energia dos fótons, seja o processo Auger, associado com a excitação (Auger ressonante) ou ionização (Auger normal) de um elétron de camada interna.

Comparando-se os resultados obtidos neste trabalho para o PMMA com os obtidos por fótons, conclui-se que este polímero 
apresenta um padrão de fragmentação bastante semelhante, quando bombardeado por estas diferentes fontes, principalmente no que se refere aos íons com massas inferiores a 60 u.m.a.. Em termos de intensidade relativa, os dados com elétrons e fótons apresentam também uma excelente concordância. Como uma energia de $1,2 \mathrm{keV}$ do feixe de elétrons é suficiente para excitar as bordas $1 \mathrm{~s}$ do carbono e 1s do oxigênio, esta semelhança não chega a ser inesperada. Estudos de dessorção iônica com variação da energia do feixe de elétrons foram realizados e estão discutidos na referência 18 .

Na Figura 5, apresentamos o espectro de massa do PVC, medido para uma energia do feixe de elétrons de $1,2 \mathrm{keV}$. Observamos a presença de um pico na relação massa/carga 35, correspondendo ao íon ${ }^{35} \mathrm{Cl}^{+}$. Outros fragmentos também podem ser observados, tais como: $\mathrm{H}^{+}, \mathrm{H}_{2}^{+}, \mathrm{H}_{3}^{+}, \mathrm{CH}_{3}^{+}, \mathrm{C}_{2} \mathrm{H}_{3}^{+}, \mathrm{C}_{2} \mathrm{H}_{5}^{+} \mathrm{e} \mathrm{C}_{3} \mathrm{H}_{7}^{+}$. As intensidades dos íons $\mathrm{H}^{+} \mathrm{e} \mathrm{CH}_{3}{ }^{+}$novamente dominam o espectro. No PVC, entretanto, observa-se uma maior contribuição dos íons $\mathrm{H}_{2}^{+} \mathrm{e}_{3}^{+}$. Um espectro cobrindo um intervalo de massa superior a 50 u.m.a. (não apresentado), apresenta outros íons característicos do PVC, a saber: $\mathrm{CH}_{2} \mathrm{CHCH}_{2} \mathrm{CH}^{+}, \mathrm{CH}_{2} \mathrm{CHCl}^{+}$(monômero), $\mathrm{CH}_{2} \mathrm{CHClCH}_{2}^{+} \mathrm{e}$ $\mathrm{CH}_{2} \mathrm{CHClCH}_{2} \mathrm{CH}^{+}$. Não foi possível observar o isótopo 37 do íon ${ }^{37} \mathrm{Cl}^{+}$, porque a resolução da experiência não era suficientemente boa. Todavia, com o emprego de um novo canhão de elétrons recentemente adquirido em nosso laboratório e a possibilidade de usar campos de extração maiores, espera-se que a resolução melhore, pelo menos 10 vezes, e dessa forma seja possível separar isótopos, como ${ }^{35} \mathrm{Cl}^{+} \mathrm{e}^{37} \mathrm{Cl}^{+}$, não observados nas condições atuais, devido à resolução de $\mathrm{m} / \Delta \mathrm{m} \sim 20(\mathrm{~m} / \mathrm{z}=35)$.

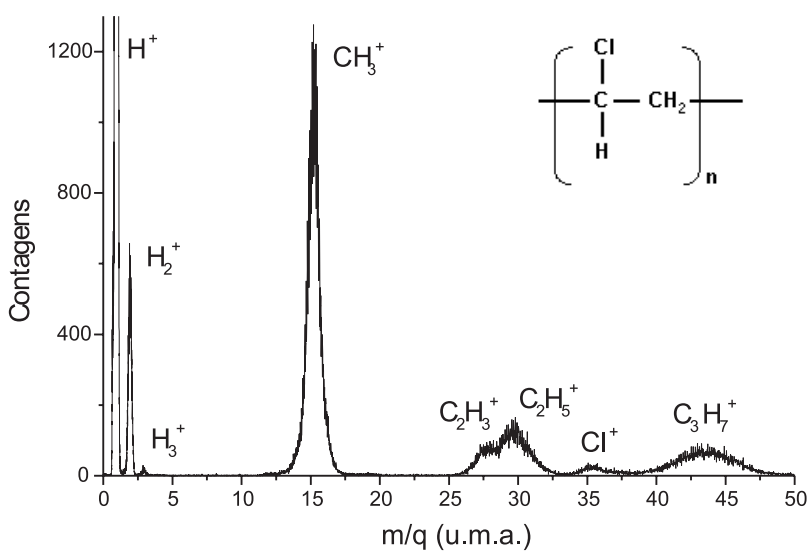

Figura 5. Espectro de massa do PVC obtido com um feixe de elétrons de $1200 \mathrm{eV}$, cobrindo um intervalo de massa de até 50 u.m.a.

É importante mencionar que durante as experiências não se observou degradação dos materiais poliméricos pela ação do feixe de elétrons, nem tampouco carregamento da superfície. Estes dois efeitos produziriam espectros TOF diferentes ou mudanças nas intensidades dos íons secundários com o tempo de irradiação. A baixa densidade de elétrons utilizada e a baixa seção de choque de dano dos elétrons explicam tais observações.

\section{CONCLUSÃO}

Com o objetivo de estudar a interação de elétrons com superfícies sólidas, desenvolveu-se um sistema experimental para estudos de dessorção iônica, utilizando um espectrômetro de massa do tipo tempo-de-vôo. Com este sistema, foram obtidos espectros de massa dos polímeros PMMA e PVC, com um feixe de elétrons pulsado de $1,2 \mathrm{keV}$. Os espectros mostraram picos comuns (por exemplo, $\mathrm{H}^{+}$, $\mathrm{H}_{2}^{+}, \mathrm{CH}_{3}^{+}$) e picos característicos de cada polímero (por exemplo, os íons $\mathrm{CHO}^{+}, \mathrm{COCH}^{+}$e $\mathrm{COCH}_{3}^{+}$no $\mathrm{PMMA} \mathrm{e} \mathrm{o}{ }^{35} \mathrm{Cl}^{+}$no PVC). Em todos eles, o íon $\mathrm{H}^{+}$foi o que apresentou a maior contagem relativa, o que indica ser a principal espécie iônica dessorvida, seguida do íon $\mathrm{CH}_{3}^{+}$. Um novo método de calibração foi empregado, permitindo uma calibração precisa dos espectros de massa apresentados. Estes resultados demonstram a eficácia do sistema experimental desenvolvido para a caracterização da superfície de polímeros e encoraja a pesquisa de outros materiais não condutores, bem como semicondutores e condutores. Cabe mencionar que, a degradação de polímeros está sendo também estudada mediante espectrometria de massa utilizando-se luz síncrotron do LNLS (Laboratório Nacional de Luz Síncrotron), de Campinas.

\section{AGRADECIMENTOS}

Os autores agradecem o apoio financeiro da FAPERJ, CNPq e FINEP. D.E.Weibel agradece à FAPERJ e à CAPES pelo apoio financeiro. Os autores gostariam ainda de expressar sua gratidão à Profa. E. E. Monteiro, do Instituto de Macromoléculas da UFRJ, pelo fornecimento das amostras de PMMA e PVC.

\section{REFERÊNCIAS}

1. Bishop, H. E. Em Methods of Surface Analysis; Walls, J. M., ed.; Cambridge University Press; Cambridge, 1989, p. 87.

2. Dreisewerd, K.; Schurenberg, M.; Karas, M.; Hillenkamp, F.; Int. J. Mass Spectrom. Ion Processes 1995, 141, 127.

3. Levis, R. J.; Annu. Rev. Phys. Chem. 1994, 45, 483.

4. Spengler, B.; Hubert, M.; J. Am. Soc. Mass Spectrom. 2002, 13, 735.

5. Benninghoven, A.; Surf. Sci. 1994, 299/300, 246.

6. Stapel, D.; Thiemann, M.; Benninghoven, A.; Surf. Sci. 2000, 158, 362.

7. Vickerman, J. C.; Briggs, D.; TOF-SIMS Surface Analysis by Mass Spectrometry; Surface Spectra Ltd: Chinchester; IM Publications, 2001

8. Rading, D.; Liebing, V.; Becker, G.; Fuchs, H.; Benninghoven, A.; J. Vac. Sci. Technol., A 1998, 16, 3449.

9. Gilmore, I. S.; Seah M. P.; Appl. Surf. Sci. 2002, 187, 89.

10. Pacholski, M. L.; Winograd N.; Chem. Rev. 1999, 99, 2977.

11. Burns, A. R.; Stechel, E. B.; Jennison, D. R.; Desorption Induced by Electronic Transition, DIET V; Spriger Series in Surface Sciences; Springer-Verlag: Berlin, Heidelberg, 1993 e edições posteriores.

12. Ishikawa, K.; Yoshimura, M.; Ueda, K.; Sakai, Y.; Rev. Sci. Instrum. 1997, $68,4103$.

13. Ishikawa, K.; Ueda, K.; Yoshimura, M.; Surf. Sci. 1999, 435, 244.

14. Ueda, K.; Ishikawa, K.; Ogai, K.; Surf. Sci. 2001, 493, 138.

15. Wollersheim, O.; Zumaqué, H.; Hormes, J.; Kadereit, D.; Langen, J.; Haubling, L.; Hoessel, P.; Hoffmann, G.; Nucl. Instrum. Methods Phys. Res., Sect. B 1995, 97, 273.

16. Zhang, Y.; Katoh, T.; Jpn. J. Appl. Phys. 1996, L186, 35.

17. Ehrfeld, W.; Münchmeyer, D.; Nucl. Instrum. Methods Phys. Res., Sect. A 1991, 303, 523.

18. Rocco, M. L. M.; Weibel, D. E.; Pontes, F. C.; Pinho, R. R.; Faraudo, G. S.; de Souza, G. G. B.; Polym. Degrad. Stab. 2003, 80, 263.

19. Maciel, J. B.; Morikawa, E.; Souza, G. G. B. de; Synchrotron Radiation Instrumentation, National Conference, AIP Conf. Proc. 417, 1997.

20. Dahl, D. A.; Simion 3D Version 6.0 User's Manual, Idaho National Engineering Laboratory, Ion Source Software, 1995.

21. Rocco, M. L. M.; Souza, G. G. B. de; Weibel, D. E.; Rev. Sci. Instrum. 2001, 72, 2827.

22. Cardoso, A. S.; Pontes, F. C.; Souza, G. G. B. de; Mundim, M. S. P.; Quim. Nova 2001, 24, 315.

23. Tinone, M. C. K.; Tanaka, K.; Maruyama, J.; Ueno, N.; Imamura, M.; Matsubayashi, N.; J. Chem. Phys. 1994, 100, 5988.

24. Madey, T. E.; Ramaker, D. E.; Stockbauer, R.; Annu. Rev. Phys. Chem. 1984, 35, 215.

25. Tanaka, K.; Tinone, M. C. K.; Ikeura, H.; Sekiguchi, T.; Sekitani, T.; Rev. Sci. Instrum. 1995, 66, 1474.

26. Ueno, N.; Tanaka, K.; Jpn. J. Appl. Phys. 1997, 36, 7605.

27. Fujii, K.; Tomimoto, H.; Isshiki, K.; Tooyama, M.; Sekitani, T.; Tanaka, K.; Jpn. J. Appl. Phys. 1999, 38 (Suppl. 1), 321.

28. Fujii, K.; Taga, D.; Nakashima, Y.; Waki, S.; Sardar, S. A.; Yasui, Y.; Wada, S.-i.; Sekitani, T.; Tanaka, K.; Nucl. Instrum. Methods Phys. Res., Sect. A 2001, 467, 1509. 